\title{
ACTIVIDAD SEXUAL EN CABRITOS CRIOLLOS NEUQUINOS DE LAPATAGONIA ARGENTINA
}

\author{
SEXUAL ACTIVITY IN CRIOLLO NEUQUINO MALE GOAT KIDS \\ OFPATAGONIA ARGENTINA
}

Gibbons, A., M. Cueto, M.R. Lanari y E. Domingo

Instituto Nacional de Tecnología Agropecuaria. Estación Experimental Agropecuaria Bariloche. CC 277 8400 Bariloche. Argentina. agibbons@bariloche.inta.gov.ar

\author{
Palabras clave adicionales \\ Pubertad. Caprinos. Capacidad copulatoria.
}

\section{RESUMEN}

El conocimiento sobre el inicio de la pubertad y la maduración sexual en una raza o biotipo de animal doméstico constituye una herramienta necesaria para realizar un correcto manejo reproductivo. Los machos caprinos de diferentes razas presentan variación en el inicio de su actividad reproductiva. En 33 cabritos Criollos de la provincia del Neuquén, manejados en condiciones extensivas de producción (Lat. $41^{\circ} 02^{\prime} \mathrm{S}$ Long. $70^{\circ}$ 34 ' O), durante los meses de abril a julio (180-270 días de edad) se determinó la ruptura de la membrana prepucial y la capacidad de copulación, mediante su permanencia durante 10 minutos con cuatro hembras caprinas inducidas en estro mediante tratamiento hormonal. Durante los meses de abril a julio las tasas mensuales de cabritos que presentaron ruptura de su membrana prepucial y capacidad de copulación fueron 69,$6 ; 78,3 ; 78,3$; $82,6 \%$ y 18,$7 ; 50 ; 61,1 ; 63,1 \%$ respectivamente. Para este período, los pesos vivos y circunferencias testiculares medias mensuales fueron 17,3 \pm 0,$07 ; 19,7 \pm 0,07 ; 19,2 \pm 0,06 ; 17,8 \pm 0,06$ kg y 16,5 $\pm 0,08 ; 16,7 \pm 0,08 ; 17,6 \pm 0,08 ; 17,2 \pm 0,07 \mathrm{~cm}$, respectivamente. Se determinó mensualmente que los cabritos sin membrana prepucial presentaron valores medios significativamente mayores en su peso vivo y circunferencia testicular con respecto a los cabritos con membrana prepucial. Asimismo, los cabritos con capacidad copulatoria tuvieron valores medios significativamente mayores en peso vivo y circunferencia testicular con respecto a los cabritos sin capacidad copulatoria. Se concluye que los cabritos Criollos Neuquinos presen-

Recibido: 26-1-07. Aceptado: 8-2-07.

\author{
AdDitiOnAL KEYWORDS \\ Puberty. Goat. Copulation capacity.
}

tan una ruptura progresiva de su membrana prepucial y un incremento en su capacidad copulatoria durante su primera estación reproductiva. El desarrollo sexual se presenta como un proceso gradual y estaría considerablemente asociado al peso vivo y a la circunferencia testicular.

\section{SUMMARY}

Detailed information on the onset of puberty and sexual maturation are needed for good reproductive management of domestic animals. Most breeds of goat are seasonal breeders and the age of puberty in male differ among them. The purpose of this investigation was to determine in 33 Neuquino Criollo kids, raised and managed under a extensive system (Lat. $41^{\circ} 02^{\prime} \mathrm{S}$ Long. $70^{\circ}$ 34 ' W) during April to July (180-270 days of age), the development of break down prepuce membrane and copulation capacity exposed to four females in hormonally induced estrus, for 10 minutes. The respective month rates non prepuce membrane and sexual copulation were $69.6,78.3,78.3$, $82.6 \%$ and $18.7,50,61.1,63.1 \%$ during April, May, June and July. For these months the live weight and testicular circumference means were $17.3 \pm$ $0.07,19.7 \pm 0.07,19.2 \pm 0.06,17.8 \pm 0.06 \mathrm{~kg}$ and $16.5 \pm 0.08,16.7 \pm 0.08,17.6 \pm 0.08,17.2 \pm 0.07$ $\mathrm{cm}$, respectively. The relation between testicular circumference and live weight in kids with or without prepuce membrane (April to July) and with or non-copulatory capacity (May to July) 
determined that kids without prepuce membrane had significantly higher means in live weight and testicular circumference than those with it. Also kids with copulation capacity had significantly higher means in live weight and testicular circumference than those with non-copulation capacity. In conclusion, Neuquino Criollo kids had a progressive lost of their prepuce membrane and increasing copulation capacity during their first breeding season. Sexual development of kids appears to be closely associated with live weight and testicular circumference.

\section{INTRODUCCIÓN}

La pubertad se define como la presencia de signos internos y externos de maduración sexual y se manifiesta por medio de la capacidad que adquieren los animales jóvenes para reproducirse. A nivel mundial, se han registrado variaciones en la manifestación de la pubertad en los machos caprinos, dependiendo de los factores genéticos y ambientales y sus interacciones (Mandani et al., 1988). En las cabras Criollas Neuquinas de la Patagonia, la actividad reproductiva se presenta en el otoño; durante la primavera y el verano se presentan naturalmente las mejores condiciones nutricionales, incidiendo en el desarrollo de la madurez sexual de las crías (Lanari et al., 2000). La pubertad marca el inicio de la etapa reproductiva de los animales, por lo que su determinación aporta la información de referencia para aplicar medidas de manejo para cada sistema de producción animal. Debido a los múltiples factores que inciden sobre el inicio de la actividad reproductiva, es necesario evaluarla para cada raza o biotipo en su medio ambiente. En base a la ausencia de información de referencia en machos caprinos Criollos del norte del Neuquén sobre la manifestación de la pubertad, se realizó el presente estudio cuyo objetivo fue determinar durante el primer otoño de vida, la presentación de la ruptura de la membrana prepucial del pene, la capacidad de copulación y la relación con el peso vivo y el diámetro testicular.

\section{MATERIAL Y MÉTODOS}

Un total de 33 cabritos Criollos del Neuquén fueron evaluados en condiciones extensivas de producción, en el área de secano del norte de la Patagonia (Lat. $41^{\circ} 02^{\prime}$ S Long. $70^{\circ} 34^{\prime} \mathrm{O}$ ). A partir de abril y con una edad de $180 \pm 5$ días y hasta una edad de 270 \pm 5 días en el mes de julio, se determinó mensualmente el peso vivo, la presencia de membrana prepucial, circunferencia máxima testicular y la capacidad de copulación. Esta última evaluación se realizó mediante la introducción de cada cabrito en corrales (25 metros cuadrados) con la presencia de cuatro cabras en estro inducido, mediante la aplicación i.m. de $1 \mathrm{mg}$ de cipionato de estradiol (ECP Estradiol, Lab Konig, Argentina), 48 horas antes de la evaluación. Durante 10 minutos se registró la capacidad copulatoria registrando la monta con eyaculación.

Los valores medios mensuales de circunferencia testicular y peso vivo en relación a la ruptura de la membrana prepucial y a la capacidad copulatoria fueron estadísticamente analizados mediante un análisis de varianza utilizando el procedimiento PROC GLM del programa SAS (1989) para un nivel de significación $\mathrm{p}<0,05$.

\section{RESULTADOSYDISCUSIÓN}

Las respectivas tasas mensuales de cabritos que presentaron ruptura de su membrana prepucial y capacidad de copulación fueron 69,$6 ; 78,3 ; 78,3 ; 82,6 \%$ y 18,$7 ; 50,0$; 61,$1 ; 63,1 \%$ durante los meses de abril a julio. Para este período, los pesos vivos y circunferencias testiculares medias mensuales fueron $17,3 \pm 0,07 ; 19,7 \pm 0,07 ; 19,2 \pm 0,06$; $17,8 \pm 0,06 \mathrm{~kg}$ y $16,5 \pm 0,08 ; 16,7 \pm 0,08 ; 17,6$ $\pm 0,08 ; 17,2 \pm 0,07 \mathrm{~cm}$, respectivamente. La relación entre circunferencia testicular y peso vivo en cabritos con o sin membrana prepucial (abril a julio) y con y sin capacidad de copulación (mayo a julio) determinó que los cabritos sin membrana prepucial pre- 


\section{ACTIVIDAD SEXUAL EN CABRITOS CRIOLLOS NEUQUINOS DE LA PATAGONIA}

Tabla I. Circunferencia testicular y peso vivo mensual de abril a julio en cabritos criollos con y sin membrana prepucial (media \pm ESM). (Testicular circumference and monthly body weight from April to July in criollo male goat kids with and without prepucial membrane (mean \pm SEM)).

\begin{tabular}{llcccc}
\hline Parámetro & Variable de clasificación & \multicolumn{3}{c}{ Determinaciones mensuales } \\
& & abril & mayo & junio & julio \\
\hline CT $(\mathrm{cm})$ & Con membrana prepucia & $12,6 \pm 0,1$ & $12,2 \pm 0,1$ & $12,8 \pm 0,2$ & $12,9 \pm 0,3$ \\
& Sin membrana prepucial & $18,2 \pm 0,1$ & $17,9 \pm 0,1$ & $19,2 \pm 0,1$ & $17,9 \pm 0,1$ \\
& Significación & $p<0,01$ & $p<0,01$ & $p<0,01$ & $p<0,01$ \\
& & & & & \\
Peso vivo $(\mathrm{kg})$ & Con membrana prepucial & $13,8 \pm 0,1$ & $16,7 \pm 0,2$ & $16,3 \pm 0,1$ & $15,5 \pm 0,08$ \\
& Sin membrana prepucial & $18,9 \pm 0,1$ & $20,5 \pm 0,08$ & $19,9 \pm 0,08$ & $18,4 \pm 0,07$ \\
& Significación & $p<0,01$ & $p<0,01$ & $p<0,01$ & $p<0,05$ \\
\hline \multirow{2}{*}{ CT= Circunferencia testicular. } & & & & \\
\end{tabular}

sentaron valores medios significativamente mayores en su peso vivo y circunferencia testicular con respecto a los cabritos con membrana prepucial $(\mathrm{p}<0,05)$ (tabla I). Asimismo, los cabritos con capacidad copulatoria tuvieron valores medios significativamente mayores en peso vivo y circunferencia testicular con respecto a los cabritos sin capacidad copulatoria $(\mathrm{p}<0,05)$ (tabla II).

En este estudio el $50 \%$ de los cabritos Criollos del Neuquén presentaron capacidad copulatoria a los 7 meses de edad y con un $35 \%$ de su peso adulto. Según las razas se presentan variaciones en la edad media en que los cabritos adquieren capacidad reproductiva, pudiendo variar entre $\operatorname{los} 4 \mathrm{a}$ 8 meses (Nishimura et al., 2000). En ovinos, Dyrmundsson y Less (1972) determinaron que los corderos de raza Clun Forest pueden alcanzar la pubertad cuando logran un peso corporal del $35-45 \%$ del peso adulto.

Las diferencias mensuales en el tamaño testicular y el peso corporal entre los animales con y sin ruptura de la membrana prepucial señalan la importancia que tiene el desarrollo gonadal y corporal sobre la liberación del pene para lograr la erección y por

Tabla II. Circunferencia testicular y peso vivo mensual de mayo a julio en cabritos criollos con y sin capacidad copulatoria (media $\pm E S M$ ). (Testicular circumference and monthly body weight from May to July in Criollo male goat kids with and without copulation capacity (mean \pm SEM)).

\begin{tabular}{llccc}
\hline Parámetro & Variable de clasificación & \multicolumn{2}{c}{$\begin{array}{c}\text { Determinaciones mensuales } \\
\text { Junio }\end{array}$} & Julio \\
\hline CT $(\mathrm{cm})$ & Con capacidad copulatoria & $19,7 \pm 0,1$ & $20,3 \pm 0,1$ & $19,2 \pm 0,1$ \\
& Sin capacidad copulatoria & $14,8 \pm 0,1$ & $15,0 \pm 0,1$ & $14,5 \pm 0,1$ \\
& Significación & $p<0,01$ & $p<0,01$ & $p<0,01$ \\
& & & & $19,5 \pm 0,08$ \\
Peso vivo $(\mathrm{kg})$ & Con capacidad copulatoria & $21,6 \pm 0,1$ & $21,2 \pm 0,1$ & $15,7 \pm 0,1$ \\
& Sin capacidad copulatoria & $18,4 \pm 0,1$ & $17,3 \pm 0,1$ & $p<0,01$ \\
& Significación & $p<0,05$ & $p<0,01$ & $p$ \\
\hline
\end{tabular}

$\mathrm{CT}=$ Circunferencia testicular. 


\section{GIBBONS, CUETO, LANARIYDOMINGO}

ende la capacidad copulatoria. La nutrición es un factor de alta incidencia sobre el desarrollo general del individuo, su crecimiento corporal y el desarrollo de sus órganos reproductivos y por ende en el comienzo de su pubertad (Dowing, 1980).

Según las observaciones realizadas el desarrollo de la capacidad reproductiva es un proceso gradual en el cual el peso corporal sería mejor indicador que la edad para determinar la pubertad en los cabritos Criollos Neuquinos, en coincidencia con otros autores (ovino: Dyrmundsson, 1973 y caprino: Belibasaki y Kouimtzis, 2000).

Sin embargo, no solamente hay que tener en consideración el grado de crecimien-

\section{BIBLIOGRAFÍA}

Belibasaki, S. and S. Kouimtzis. 2000. Sexual activity and body and testis growth in prepubertal ram lambs of Friesland, Chios, Karagouniki and Serres dairy sheep in Greece. Small Rum. Res., 37: 109-113.

Dowing, J.M. 1980. Studies on the effects of date of birth and plane of nutrition on attainment of puberty and reproductive performance in Clun Forest ewe lambs. PhD Thesis. University of Wales.

Dyrmundsson, O.R. and J.L. Less. 1972. Attainment of puberty and reproductive performance in Clun Forest ewe lambs. J. Agr. Sci., 78: 39.

Dyrmundsson, O.R. 1973. Puberty and early reproductive performance in sheep. Ewe lambs. to corporal y tamaño testicular, sino que además, la edad mínima establece un límite por debajo de la cual no se expresaría la pubertad, independientemente del peso vivo y testicular (Dyrmundsson y Less, 1972). Por lo tanto, se debe considerar que el peso vivo y la edad presentan valores mínimos necesarios para que una determinada raza sea susceptible de alcanzar la pubertad.

Se concluye que los cabritos Criollos Neuquinos presentan una ruptura progresiva de su membrana prepucial y un incremento en su capacidad copulatoria durante su primera estación reproductiva, estando considerablemente asociada a su peso vivo y circunferencia testicular.

Anim. Breed. Abstr., 41: 273.

Lanari, M., M. Pérez Centeno, E. Domingo y C. Robles. 2000. Caracterización de caprinos CrioIlos del Norte de Neuquén (Patagonia, Argentina). $5^{\circ}$ Congreso Iberoamericano de Razas Autóctonas y Criollas. La Habana. Cuba.

Mandani, M.O.K. and M.S. Rahal. 1988. Puberty in Libyan male goats. Anim. Reprod. Sci., 17: 207216.

Nishimura, S., K. Okano, K. Yasukouchi, T. Gotoh, S. Tabata and H. Iwamoto. 2000. Testis developments and puberty in the male Tokara (Japanese native) goat. Anim. Reprod. Sci., 64: 127-131. SAS. 1989. User's Guide: Statistics, Version 6, SAS Institute Inc., Cary, NC.

Archivos de zootecnia vol. 58, núm. 221, p. 132. 